\title{
Thermodynamics of fractal signals based on wavelet analysis: application to fully developed turbulence data and DNA sequences
}

\author{
A. Arneodo ${ }^{\mathrm{a}, *}$, B. Audit ${ }^{\mathrm{a}}$, E. Bacry ${ }^{\mathrm{b}}$, S. Manneville ${ }^{\mathrm{a}}$, J.F. Muzy $^{\mathrm{a}}$, \\ S.G. Roux ${ }^{\text {a }}$ \\ ${ }^{a}$ Centre de Recherche Paul Pascal, Avenue Schweitzer, 33600 Pessac, France \\ ${ }^{\mathrm{b}}$ Centre de Mathématiques Appliquées, Ecole Polytechnique, 91128 Palaiseau, France
}

\begin{abstract}
We use the continuous wavelet transform to generalize the multifractal formalism to fractal functions. We report the results of recent applications of the so-called wavelet transform modulus maxima (WTMM) method to fully developed turbulence data and DNA sequences. We conclude by briefly describing some works currently under progress, which are likely to be the guidelines for future research. (c) 1998 Elsevier Science B.V. All rights reserved
\end{abstract}

\section{From global to local characterization of the regularity of fractal functions}

In many situations in physics as well as in some applied sciences, one is faced to the problem of characterizing very irregular functions [1-11]. The examples range from plots of various kinds of random walks, e.g. Brownian signals $[12,13]$, to financial timeseries [14-16], to geological shapes [1,9,17], to medical time-series [18], to interfaces developing in far from equilibrium growth processes [4,6,11], to turbulent velocity signals $[10,19,20]$ and to "DNA walks" coding nucleotide sequences $[21,22]$. These functions can be qualified as fractal functions $[1,13,23-25]$ whenever their graphs are fractal sets in $\mathbb{R}^{2}$ (for our purpose here we will only consider functions from $\mathbb{R}$ to $\mathbb{R})$. They are commonly called self-affine functions since their graphs are similar to themselves when transformed by anisotropic dilations, i.e., when shrinking along the $\mathrm{x}$-axis by a factor $\lambda$ followed by a rescaling of the increments of the function by a different factor $\lambda^{-H}: \forall x_{0} \in \mathbb{R}, \exists H \in \mathbb{R}$ such that for any $\lambda>0$, one has

$$
f\left(x_{0}+\lambda x\right)-f\left(x_{0}\right) \simeq \lambda^{H}\left(f\left(x_{0}+x\right)-f\left(x_{0}\right)\right) .
$$

* Corresponding author. Tel.: +33 556845665; fax: +33556845600; e-mail: arneodo@crpp.u-bordeaux.fr. 
If $f$ is a stochastic process, this identity holds in law for fixed $\lambda$ and $x_{0}$. The exponent $H$ is called the roughness or Hurst exponent $[1,3,6]$. The graph of $f$ is self-similar if it is invariant under some isotropic dilations, i.e., when the Hurst exponent $H=1$. Let us note that if $H<1$, then $f$ is not differentiable and the smaller the exponent $H$, the more singular $f$. Thus the Hurst exponent provides indication of how globally irregular the function $f$ is.

Different methods [6] (e.g., height-height correlation function, variance and power spectral methods) have been used to estimate the roughness exponent which is supposed to be related to the fractal dimension $D_{\mathrm{F}}=2-H$ of the graph of the considered function. But beyond some practical algorithmic limitations, there exists a more fundamental intrinsic insufficiency in the fractal dimension and/or roughness exponent measurement in the sense that $D_{\mathrm{F}}$ as well as $H$ are global quantities that do not account for possible fluctuations (from point to point) in the local regularity of $f$. To describe these nonhomogeneous functions, one thus needs to change slightly the definition of the Hurst regularity so that it becomes a local quantity [26-29]:

$$
\left|f\left(x_{0}+l\right)-f\left(x_{0}\right)\right| \sim l^{h\left(x_{0}\right)} .
$$

This "local roughness exponent" $h\left(x_{0}\right)$ corresponds to the Hölder exponent of $f$ at the point $x_{0}$ when $h\left(x_{0}\right)<1$. The Hölder exponent characterizes the strength of the singularity of $f$ at the point $x_{0}$ and is defined as the largest exponent such that there exists a polynomial $P_{n}\left(x-x_{0}\right)$ of order $n$ satisfying [30-33]

$$
\left|f(x)-P_{n}\left(x-x_{0}\right)\right| \leqslant C\left|x-x_{0}\right|^{h},
$$

for $x$ in the neighborhood of $x_{0}$. If $\left.h\left(x_{0}\right) \in\right] n, n+1[$, one can easily prove that $f$ is $n$ times, but not $n+1$ times differentiable at the point $x_{0}$. The polynomial $P_{n}\left(x-x_{0}\right)$ corresponds to the Taylor series of $f$ around $x=x_{0}$ up to the order $n$. Thus the higher the exponent $h\left(x_{0}\right)$, the more regular the function $f$ at the point $x_{0}$.

\section{Statistical analysis of the regularity of fractal functions: the multifractal formalism}

The multifractal formalism [34-43] has been originally established to account for the statistical scaling properties of singular measures arising in various situations in physics, chemistry, geology or biology. Notable examples include the invariant probability distribution on a strange attractor $[36,38,43]$, the distribution of voltage drops across a random resistor network $[2,3,44]$, the distribution of growth probabilities on the boundary of a diffusion-limited aggregate $[3,4,45]$ and the spatial distribution of dissipative regions in a turbulent flow [41,46-48]. This formalism lies upon the determination of the so-called $f(\alpha)$ singularity spectrum [36] which characterizes the relative contribution of each singularity of the measure: let $S_{\alpha}$ be the subset of points $x$ where the measure of an $\varepsilon$-box $B_{x}(\varepsilon)$, centered at $x$, scales like $\mu\left(B_{x}(\varepsilon)\right) \sim \varepsilon^{\alpha}$ in the limit $\varepsilon \rightarrow 0^{+}$, then by definition, $f(\alpha)$ is the Hausdorff dimension of $S_{\alpha}: f(\alpha)=\operatorname{dim}_{\mathrm{H}}\left(S_{\alpha}\right)$. 
Actually, there exists a deep analogy that links the multifractal formalism with that of statistical thermodynamics [49-51]. This analogy provides a natural connection between the $f(\alpha)$ spectrum and a directly observable spectrum $\tau(q)$ defined from the power-law behavior, in the limit $\varepsilon \rightarrow 0^{+}$, of the partition function [36]:

$$
Z_{q}(\varepsilon)=\sum_{i} \mu\left(B_{i}(\varepsilon)\right)^{q} \sim \varepsilon^{\tau(q)},
$$

where the sum is taken over a partition of the support of the singular measure into boxes of size $\varepsilon$. The variables $q$ and $\tau(q)$ play the same role as the inverse of temperature and the free energy in thermodynamics while the Legendre transform,

$$
f(\alpha)=\min _{q}(q \alpha-\tau(q)),
$$

indicates that instead of energy and entropy, we have $\alpha$ and $f(\alpha)$ as the thermodynamical variables conjugate to $q$ and $\tau(q)$ [36-39,52]. Let us mention that the so-called generalized fractal dimensions $D_{q}$ [53-57] are nothing else than $D_{q}=\tau(q) /(q-1)$.

In the past few years, the multifractal approach has proved particularly fruitful in many contexts. However, as pointed out previously, in physics and other applied sciences, fractals appear not only as singular measures, but also as singular functions. There have been several attempts to extend the concept of multifractal to singular functions [26,27]. In the context of fully developed turbulence [58], the intermittent character of turbulent velocity signals was investigated by calculating the moments of the probability density function (pdf) of (longitudinal) velocity increments $\delta v_{l}(x)=v(x+l)-v(x)$, over inertial separation $[26,58]$ :

$$
S_{p}(l)=\left\langle\delta v_{l}^{p}\right\rangle \sim l^{\zeta_{p}} .
$$

By Legendre transforming the scaling exponents $\zeta_{p}$ of these structure functions (SF's) of order $p$ [59], one gets the Hausdorff dimension $D(h)$ of the subset of $\mathbb{R}$ for which velocity increments behave as $\delta v_{l} \sim l^{h}[26]$ :

$$
D(h)=\min _{p}\left(p h-\zeta_{p}+1\right) .
$$

In a more general context, $D(h)$ will be the spectrum of Hölder exponents for the singular signal under study and thus will have a similar status than the $f(\alpha)$ singularity spectrum for singular measures (Eq. (5)). However, as discussed in the next section, there are some fundamental limitations to the SF approach which intrinsically fails to fully characterize the $D(h)$ singularity spectrum [60].

\section{A thermodynamics of fractal signals based on wavelet analysis}

The wavelet transform (WT) is a mathematical technique introduced in signal analysis in the early eighties [61,62]. Since then, it has been the subject of considerable theoretical developments and practical applications in a wide variety of fields 
[63-73]. The WT has been recently emphasized as a mathematical microscope that is well adapted to reveal the hierarchy that governs the spatial distribution of the singularities of multifractal measures [28,29,71,73-83]. What makes the WT of fundamental use in the present study is that its singularity scanning ability equally applies to singular functions than to singular measures $[28,29,71]$.

\subsection{Singularity detection and processing with wavelets}

The WT is a space-scale analysis which consists in expanding signals in terms of wavelets which are constructed from a single function, the analyzing wavelet $\psi$, by means of translations and dilations. The continuous WT of a real-valued function $f$ is defined as $[61,62]$

$$
T_{\psi}[f]\left(x_{0}, a\right)=\frac{1}{a} \int_{-\infty}^{+\infty} f(x) \psi\left(\frac{x-x_{0}}{a}\right) \mathrm{d} x,
$$

where $x_{0}$ is the space parameter and $a(>0)$ the scale parameter. The analyzing wavelet $\psi$ is generally chosen to be well localized in both space and frequency. Usually, $\psi$ is required to be of zero mean for the WT to be invertible. The main advantage of using the WT for analyzing the regularity of a function, is its ability to be blind to smooth (polynomial) behavior by an appropriate choice of the analyzing wavelet $\psi$. Indeed, let us assume that according to Eq. (3), $f$ has a local scaling exponent $h\left(x_{0}\right)$ at the point $x_{0}$; then one can easily prove that the local behavior of $f$ is mirrored by the WT which locally behaves like [30-33]:

$$
T_{\psi}[f]\left(x_{0}, a\right) \sim a^{h\left(x_{0}\right)}, \quad a \rightarrow 0^{+},
$$

provided $n_{\psi}>h\left(x_{0}\right)$, where $n_{\psi}$ is the number of vanishing moments of $\psi$, i.e.

$$
\int_{-\infty}^{+\infty} x^{m} \psi(x) \mathrm{d} x=0, \quad \forall m, 0 \leqslant m<n_{\psi} .
$$

Thus, one can extract the exponent $h\left(x_{0}\right)$ from a log-log plot of the WT amplitude versus the scale $a$. Throughout this paper, we will mainly use the class of analyzing wavelets defined by the successive derivatives of the Gaussian function $[28,29,84]$.

In the case of isolated singular behavior, one can therefore use Eq. (9) to determine numerically the corresponding Hölder exponent. The situation is somewhat more intricate when investigating fractal signals due to the existence of a hierarchical distribution of singularities [28,29,33,74,85,86]. Locally, the Hölder exponent $h\left(x_{0}\right)$ is governed by the singularities which accumulate at $x_{0}$. This results in unavoidable oscillations around the expected power-law behavior of the WT amplitude [28,29,85-87]. Therefore, the exact determination of $h$ from $\log -\log$ plots on a finite range of scales is somewhat uncertain $[88,89]$. Of course there have been many attempts to circumvent these difficulties $[86,90]$. Nevertheless, there exist fundamental limitations (which are not intrinsic 
to the WT technique) to the local measurement of Hölder exponents of fractal functions. Consequently, the determination of statistical quantities like the $D(h)$ singularity spectrum, requires a method which is more feasible and more appropriate than the histogram method experienced in Refs. [88,89] and which amounts to a systematic investigation of the WT local scaling behavior.

\subsection{The wavelet transform modulus maxima method}

A natural way of performing a multifractal analysis of fractal functions consists in generalizing the "classical" multifractal formalism [36] using wavelets instead of boxes. By taking advantage of the freedom in the choice of the "generalized oscillating boxes" that are the wavelets, one can hope to get rid of possible smooth behavior that could mask singularities or perturb the estimation of their strength $h$. But the major difficulty with respect to box-counting techniques [57,84,91] for singular measures, consists in defining a covering of the support of the singular part of the function with our set of wavelets of different sizes. A simple method would thus rely on the definition of the following partition function in terms of WT coefficients [28,29,74]:

$$
Z(q, a)=\int\left|T_{\psi}[f](x, a)\right|^{q} \mathrm{~d} x
$$

where $q \in \mathbb{R}$. This method based on a continuous covering of the real line would be a rather naive generalization of box-counting algorithms since nothing prevents the WT from vanishing at some point $\left(x_{0}, a\right)$ of the space-scale half-plane. The partition function may then diverge for $q \leqslant-1$.

The wavelet transform modulus maxima (WTMM) method [28,29,33,60,92] consists in building a partition function from the modulus maxima of the WT. The maxima are defined, at each scale $a$, as the local maxima of $\left|T_{\psi}[f](x, a)\right|$ considered as a function of $x$. As illustrated in Fig. 1c, these WTMM are disposed on connected curves called maxima lines. Let us define $\mathscr{L}\left(a_{0}\right)$ as the set of all the maxima lines that exist at the scale $a_{0}$ and which contain maxima at any scale $a \leqslant a_{0}$. An important feature of these maxima lines is that, each time the analyzed signal has a local Hölder exponent $h\left(x_{0}\right)<n_{\psi}$, there is at least one maxima line pointing towards $x_{0}$ along which Eq. (9) holds [32,33]. In the case of fractal signals, we thus expect that the number of maxima lines will diverge in the limit $a \rightarrow 0^{+}$. In fact, as emphasized in Refs. [28,29,92], the branching structure of the WTMM skeleton in the $(x, a)$ half-plane enlightens the hierarchical organization of the singularities. The WTMM method consists in taking advantage of the space-scale partitioning given by this skeleton to define the following partition function [28,29,33,60,92]:

$$
Z(q, a)=\sum_{l \in \mathscr{L}(a)}\left(\sup _{\substack{\left(x, a^{\prime}\right) \in l \\ a^{\prime} \leqslant a}}\left|T_{\psi}[f]\left(x, a^{\prime}\right)\right|\right)^{q} .
$$



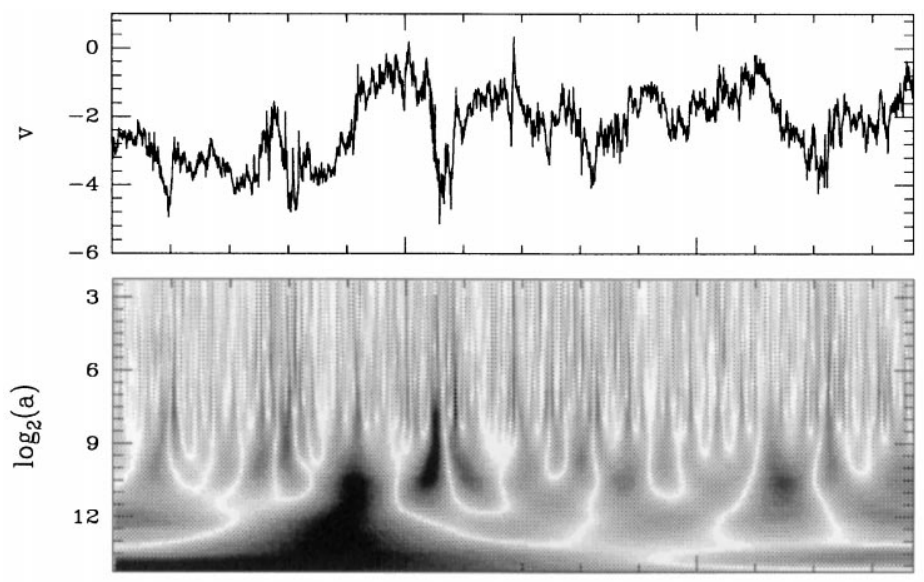

(b)

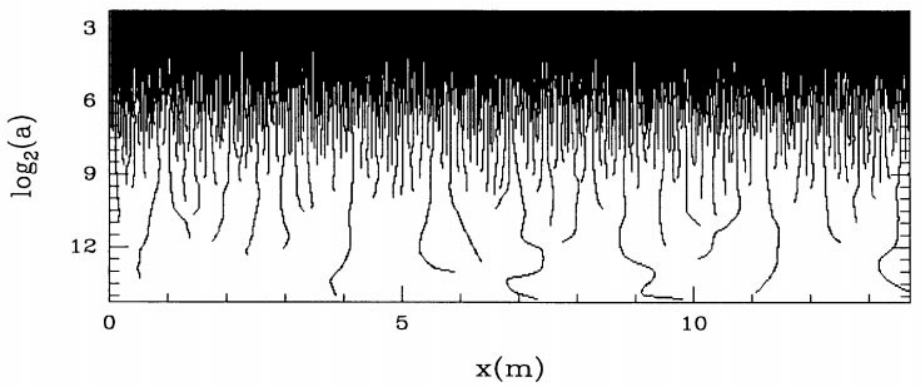

(c)

Fig. 1. Continuous WT of fully developed turbulence from wind tunnel data. (a) The turbulent velocity signal over about two integral scales. (b) WT of the turbulent signal; the amplitude is coded, independently at each scale $a$, using 32 grey levels from white $\left(\left|T_{\psi}[v](x, a)\right|=0\right)$ to black $\left(\max _{x}\left|T_{\psi}[v](x, a)\right|\right)$. (c) WT skeleton defined by the set of all the maxima lines. In (b) and (c), the small scales are at the top. The analyzing wavelet is a first-order $\left(n_{\psi}=1\right)$ compactly supported wavelet (see Fig. 2.18 in Ref. [98]).

The sup in Eq. (12) can be regarded as a way to define a scale adaptative partition which will make the computation of the partition function scaling exponents

$$
Z(q, a) \sim a^{\tau(q)}, \quad a \rightarrow 0^{+},
$$

more robust specially for $q<0$. Then, by using both the WT scaling behavior (Eq. (9)) along the maxima lines and the definition of the singularity spectrum $D(h)=\operatorname{dim}_{\mathrm{H}}\{x \mid$ $h(x)=h\}$, one can show that $D(h)$ can be determined by Legendre transforming $\tau(q)$ $[33,93]$ :

$$
D(h)=\min _{q}(q h-\tau(q)) .
$$

From the properties of the Legendre transform, it is easy to convince oneself that homogeneous fractal functions that involve singularities of unique Hölder exponent $h=\partial \tau / \partial q$, are characterized by a $\tau(q)$ spectrum which is a linear function of $q$. On the contrary, a nonlinear $\tau(q)$ curve is a signature of nonhomogeneous functions that 
display multifractal properties, in the sense that the Hölder exponent $h(x)$ is a fluctuating quantity that depends upon the spatial position.

Remark. The partition function exponents $\tau(q)$ are much more than simply some intermediate quantities of a rather easy experimental access. For some specific values of $q$, they have well known meaning $[28,86]$. In full analogy with standard box-counting arguments, $-\tau(0)$ can be identified to the fractal dimension of the set of singularities of $f$. Similarly, $\tau(1)$ is related to the capacity of the graph $\mathscr{G}$ of the considered function: $d_{\mathrm{C}}(\mathscr{G})=\max (1,1-\tau(1))$. Moreover, $\tau(2)$ is related to the scaling exponent $\beta$ of the spectral density $S(k)=|\hat{f}(k)|^{2} \sim k^{-\beta}$ with $\beta=2+\tau(2)$.

\subsection{The structure function approach versus the WTMM method}

It is tempting to relate the exponents $\tau(q)$, defined from the WT partition function (Eqs. (12) and (13)), to the scaling exponents $\zeta_{p}$ of the structure functions (Eq. (6)). A simple comparison of Eqs. (7) and (14) gives immediately [28,86]:

$$
\tau(q)=\zeta_{q}-1
$$

But this relationship does not hold for all values of $q$; this results from intrinsic limitations of the SF approach [60]. As pointed out in previous studies [60,86,90], the local increment of a function can be seen as its WT computed at point $x_{0}$ and scale $l$, with the "poor man's wavelet" $\Delta(x)=\delta(x-1)-\delta(x)$. In this spirit, the SF's (Eq. (6)) are analogous to the naive partition functions defined in Eq. (11):

$$
S_{q}(l)=\int\left|\delta f_{l}(x)\right|^{q} \mathrm{~d} x .
$$

However, there are two main differences between Eqs. (12) and (16) from which result the insufficiencies of the SF method [60].

(1) The continuous integral used to define $S_{q}(l)$. Indeed, there is no reason, a priori, that the increment pdf vanishes around $\delta f_{l}=0$. Thus the SF $S_{q}(l)$ may diverge for $q<0$. Consequently, the $\zeta_{q}$ spectrum is not defined for $q<0$ and from the Legendre transform properties, only the (increasing) part of the $D(h)$ singularity spectrum corresponding to the strongest singularities is amenable to the SF approach. The first crucial advantage of the WTMM method is that the partition function (Eq. (12)) is computed using a discrete summation over the WT skeleton where the WT coefficients do not vanish (by definition of the WTMM); this keeps the calculation of $Z(q, a)$ clear of divergences for $q<0$.

(2) The poorness of the analyzing wavelet $\Delta(x)$. The second main disadvantage of the SF method is that the "poor's man wavelet" $\Delta(x)[60,86,90]$ does not satisfy the criteria to be an efficient analyzing wavelet. First $\Delta(x)$ is only orthogonal to constants but not to higher order polynomials $\left(n_{\psi}=1\right)$. This precludes the detection of singularities with $h \geqslant 1$. Thus, as demonstrated in Ref. [60], if the maximum value of $D(h)$ 
is reached for some $h \geqslant 1$, the accessible range of singularities is even more truncated to values $h<h_{\text {crit }}<1$. Moreover, $\Delta(x)$ is a singular analyzing wavelet made of two Dirac distributions and therefore it cannot generally be integrated against tempered distributions. When using Eq. (16) to study some distribution which involves singularities with $h \leqslant 0$, one is generally faced to severe instabilities in the computation of the SF's. Therefore, the range of Hölder exponents accessible to the SF method is not only limited from above $\left(h<h_{\text {crit }} \leqslant 1\right)$, but also from below $(h>0)$. Because one has the freedom to select an analyzing wavelet $\psi$ which is smooth and which has enough vanishing moments $\left(n_{\psi}>h_{\max }\right)$, the WTMM method does not possess any of these drawbacks [60].

\section{Numerical and experimental applications of the WTMM method}

The WTMM method has been tested on pedagogical examples, e.g., generalized devil's staircases, synthetic multifractal signals and fractional Brownian motions $[28,29,33,60,92]$. It has already been successfully applied to numerical and experimental data from various domains $[71,83]$. Here we will report on recent results obtained in two apparently unrelated fields, namely fully developed turbulence and DNA sequences.

\subsection{Fully developed turbulence}

It is now well-accepted $[47,58,59]$ that in the fully developed regime, a turbulent flow is likely to be in a universal state that can be experimentally characterized by statistical quantities such as the scaling exponents $\zeta_{p}$ of the longitudinal velocity structure functions or the shape of the velocity increment pdf's. For more than thirty years, one of the main features recognized experimentally, is the intermittency of small scales $[10,47,48,58,59]$; this phenomenon manifests in a nonlinear behavior of $\zeta_{p}$ that does not seem to depend on the Reynolds number $[19,20,94]$. This observation reveals some departure of the experimental data from the prediction $\zeta_{p}=p / 3$ of Kolmogorov (K41) [95] based on the assumption that, at each point of the fluid, the velocity field has the same scaling behavior $\delta v_{l}(x) \sim l^{1 / 3}$, which yields the well known $E(k) \sim k^{-5 / 3}$ energy spectrum. This nonlinear $\zeta_{p}$ spectrum actually characterizes some evolution of the velocity increment pdf from a Gaussian shape at large scales to stretched exponentials towards smaller scales in the inertial range $[20,96,97]$. Our goal here is to go beyond the SF approach (and its insufficiencies) and to use the WTMM method to achieve a multifractal analysis of single point velocity data from high Reynolds three-dimensional turbulence $[28,29,98,99]$.

The data were obtained by Gagne and collaborators in the large wind tunnel S1 of ONERA at Modane. The Taylor scale based Reynolds number is $R_{\lambda} \simeq 2000$ and the extent of the inertial range following approximately the Kolmogorov $k^{-5 / 3}$ law is about four decades (integral scale $L \simeq 7 \mathrm{~m}$, dissipation scale $\eta \simeq 0.27 \mathrm{~mm}$ ). Fig. 1 

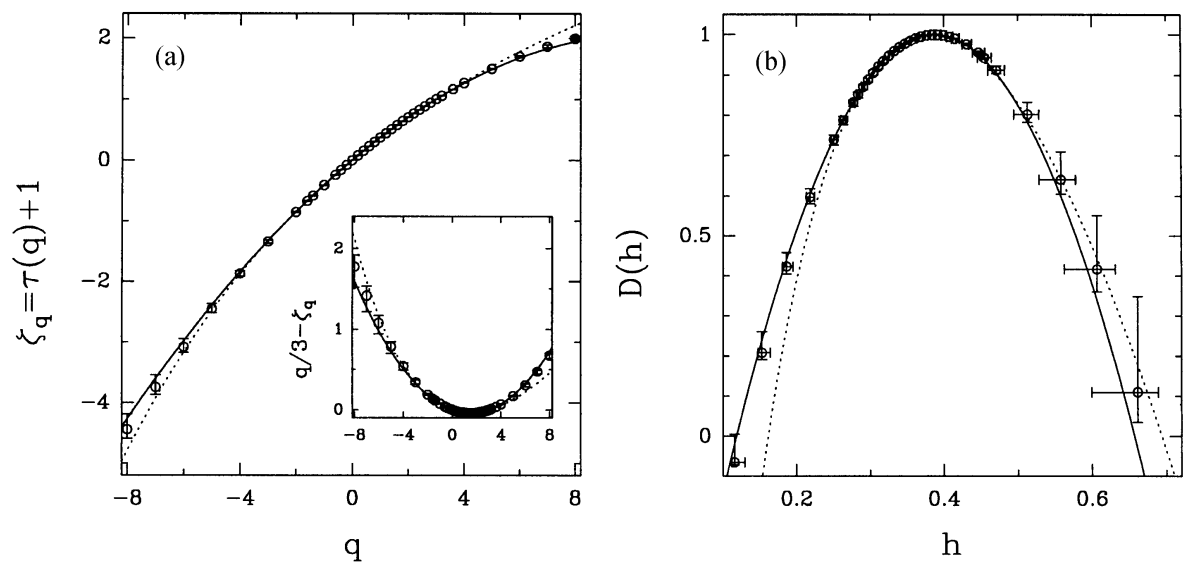

Fig. 2. WTMM measurement of the $\zeta_{q}=\tau(q)+1$ and $D(h)$ spectra of the Modane turbulent velocity signal. (a) $\zeta_{q}$ vs $q$. (b) $D(h)$ vs $h$. The solid lines correspond to a fit of the data with the theoretical quadratic spectra for log-normal random cascade processes. The dotted lines correspond to the predictions of the She and Leveque's model [106] when assuming log-Poisson statistics for the energy dissipation (see text). Same analyzing wavelet as in Fig. 1.

illustrates the WT of a sample of the (longitudinal) velocity signal of length of about two integral scales, when using the Taylor hypothesis [20,58]. In Fig. 2a are reported the results of the estimate of the $\zeta_{q}=\tau(q)+1$ scaling exponents of the partition function $Z(q, a)$ (Eq. (12)), as computed with the WTMM method for an overall statistical sample of about 28000 integral scales. As advocated by Benzi et al. $[100,101]$, we have used the extended self-similarity (ESS) concept to improve scaling: $Z(q, a) / Z(0, a) \sim(Z(3, a) / Z(0, a))^{\zeta_{q}}$ (fixing $\zeta_{3}=1$ ). Over a range of values of $q$ that extends from -8 to +8 , for which statistical convergence is achieved, the data remarkably fall on a nonlinear quadratic curve $\zeta_{q}=-m q-\sigma^{2} q^{2} / 2\left(m=-0.388, \sigma^{2}=0.036\right)$, as predicted for log-normal random cascade processes [98,102-104]. In the insert of Fig. $2 \mathrm{a}$, the deviation of the $\zeta_{q}$ 's from the K41 linear spectrum $q / 3$, is shown to be significant and quite compatible with a quadratic correction [105]. In Fig. 2a is also shown for comparison the $\zeta_{q}$ spectrum predicted by She and Leveque [106], $\zeta_{q}=q / 9+2\left[1-(2 / 3)^{q / 3}\right]$, when postulating log-Poisson statistics for the local nondimensional energy dissipation $[107,108]$ and assuming the validity of the local Kolmogorov scaling relation (K62) [102]. This last relation connects the local dissipation $\varepsilon_{l}(x)$ averaged over a domain of size $l$ and the velocity increment $\delta v_{l}(x): \varepsilon_{l}(x)={ }^{s}\left(\delta v_{l}(x)\right)^{3} / l$, where the symbol $={ }^{s}$ means that the two quantities have the same scaling laws (i.e., the scaling exponents are the same for the corresponding moments of arbitrary order). This model provides also a rather good fit of the data for $\zeta_{q}$, even though some systematic deviation becomes perceptible for $|q| \geqslant 6$. The $D(h)$ singularity spectrum obtained by Legendre transforming the experimental $\tau(q)$ spectrum is shown in Fig. 2b. Its characteristic single humped shape over a finite range of Hölder exponents, $h \in[0.12,0.68]$, is a clear signature of the multifractal nature of the turbulent signal. 
Its remarkable parabolic shape is an additional evidence for the possible relevance of log-normal random cascade processes. For $q=0$, the largest dimension is attained for singularities of exponent $h=0.39 \pm 0.01$, i.e., a value which is slightly higher than the K41 prediction $h=1 / 3$. Moreover, the corresponding maximum of the $D(h)$ curve, $D(h(q=0))=-\tau(0)=0.999 \pm 0.001$, together with the upper bound $h_{\max }=0.68<1$, strongly suggests that the turbulent velocity signal is singular everywhere. This observation is corroborated by the robustness of the $D(h)$ data with respect to changes in the choice of the analyzing wavelet: similar quantitative estimates of the $\tau(q)$ and $D(h)$ spectra are obtained when using first-order $\left(n_{\psi}=1\right)$, second-order $\left(n_{\psi}=2\right)$ and fourth-order $\left(n_{\psi}=4\right)$ analyzing wavelets [98].

From the analogy with thermodynamical potentials [28,29], it is not such a surprise that the experimental determination of the multifractal spectra $\zeta_{q}$ and $D(h)$ does not provide a selective test to discriminate between various (deterministic or random) cascade models. In order to go further in the investigation of the velocity fluctuations in turbulent flows, we have computed the pdf's of WT coefficients at different scales in the inertial range $[98,104]$. Very much like the velocity increment pdf's, these distributions are nearly centered with a shape that goes from Gaussian at large scales to more intermittent profiles with stretched exponential-like tails at smaller scales. But the wavelet theory tells us that there exists some redundancy in the continuous WT representation [61-72]. Indeed, for a given analyzing wavelet, there exists a reproducing kernel $[62,109]$ from which one can express any WT coefficient at a given point $x$ and scale $a$ as a linear combination of the neighboring WT coefficients in the space-scale half-plane. As emphasized in Refs. [32,33], a way to break free from this redundancy is to use the WTMM representation. In Fig. 3a are reported the results of the computation of the WTMM pdf's when restricting our analysis to the WT skeleton (Fig. 1c) defined by the WT maxima lines. Since by definition the WTMM are different from zero, the so-obtained pdf's decrease exponentially fast to zero at zero, which makes the estimate of the exponents $\zeta_{q}$ tractable for $q<0$ (Eq. (12)). When plotting $\ln P_{a}(\ln |T|)$ vs $\ln |T|$, one gets the remarkable result that for any scale in the inertial range, all the data points fall on a parabola, which is a strong indication that the statistics of the logarithm of the WT coefficients is definitely Gaussian with a mean and a variance that depend upon the scale parameters $[98,105]$. This implies that, along the line of Castaing et al. ansatz [96,110-116], the WTMM pdf at a given scale $a$ can be expressed in terms of the pdf at a coarser scale $a^{\prime}$ as

$$
P_{a}(T)=\int G_{a a^{\prime}}(\ln r) \frac{1}{r} P_{a^{\prime}}\left(\frac{T}{r}\right) \mathrm{d} \ln r,
$$

where $G_{a a^{\prime}}$ is a Gaussian kernel that depends upon $a$ and $a^{\prime}$ only. The term $\log$ normal self-similar cascade $[98,104,105]$ can be used since, from the above definition, one can show that there exists a decreasing sequence of scales $\left(a_{1}, \ldots, a_{n}\right)$ for which $G_{a_{n} a_{1}}=G_{a_{n} a_{n-1}} \otimes \cdots \otimes G_{a_{2} a_{1}}$, where $\otimes$ denotes the convolution product and $G_{a_{n} a_{n-1}}=G$ is a Gaussian independent of $n$. Therefore, $G_{a a^{\prime}}(\ln r)=G\left(\ln r, s\left(a, a^{\prime}\right)\right)$, 

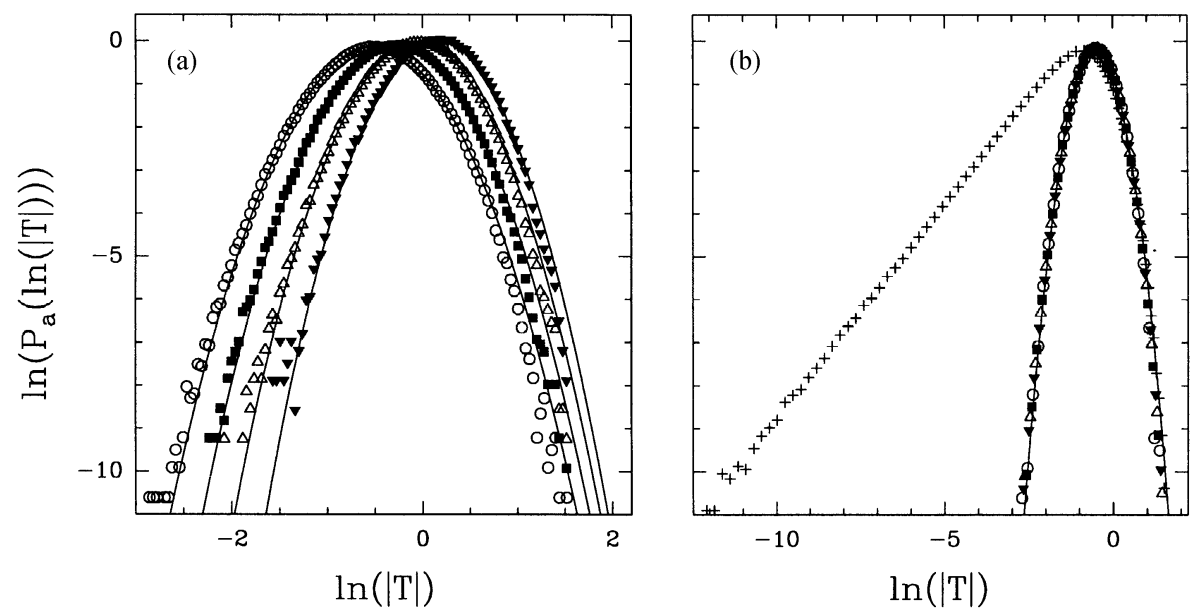

Fig. 3. Probability density functions of the WTMM of the Modane turbulent velocity signal. (a) $\ln \left(P_{a}(\ln (|T|))\right)$ vs $\ln |T|$ as computed at different scales $a=385 \eta(0), 770 \eta(\square), 1540 \eta(\triangle)$, and $3080 \eta$ $(\boldsymbol{\nabla})$. (b) The same pdf's after being transformed according to Eq. (17) with a Gaussian kernel $G_{a a^{\prime}}(x)$ with $s\left(a, a^{\prime}\right)=\left(a^{\prime-\beta}-a^{-\beta}\right) / \beta$ where $\beta=0.095$. The $(+)$ represent the corresponding velocity increment pdf. The solid lines correspond to the Gaussian approximations of these histograms. Same analyzing wavelet as in Fig. 1.

where $s\left(a, a^{\prime}\right)=s(a)-s\left(a^{\prime}\right)$ accounts for the number of steps of the cascade from scale $a^{\prime}$ to scale $a$ [116]. Using Novikov's definition [117], scale invariance amounts to write $s\left(a, a^{\prime}\right)=\ln \left(a / a^{\prime}\right)$, i.e., $s(a)=\ln a$. In Fig. $3 \mathrm{~b}$, we have succeeded in collapsing all the WTMM pdf's, computed at different scales, onto a single parabolic curve when using Eq. (17) with a Gaussian kernel $G\left(\ln r, s\left(a, a^{\prime}\right)\right)$ where $s(a)=\left(1-a^{-\beta}\right) / \beta$ with $\beta=0.095$. This is some unambiguous evidence for scale invariance breaking $[98,104,105,118]$. We have reproduced our analysis for other turbulent flows with a Reynolds number ranging from $R_{\lambda} \simeq 200$ to 3000 ; these studies corroborate the experimental fact that, with the available statistics, the various log-infinitely divisible cascade models proposed in the literature [107,108,113,117], are hardly distinguishable from their log-normal approximations, at least for the highest Reynolds numbers investigated. Moreover, when increasing $R_{\lambda}$, the exponent $\beta$ is found to decrease to zero, which suggests that scale invariance is only reached asymptotically in the limit $R_{\lambda} \rightarrow+\infty$ (in the limit $\beta \rightarrow 0, s(a)=\left(1-a^{-\beta}\right) / \beta$ reduces to $\ln a$ ). Let us note that, according to these findings, one would expect to observe some departure from scaling of the partition function $Z(q, a)$ at finite Reynolds number. Hopefully this breaking of scale invariance does not invalidate the ESS hypothesis $[98,104,105]$. This means that the WTMM estimates of the multifractal spectra $\zeta_{q}$ and $D(h)$ in Fig. 2 are likely to be valid in the infinite Reynolds number asymptotic limit. 


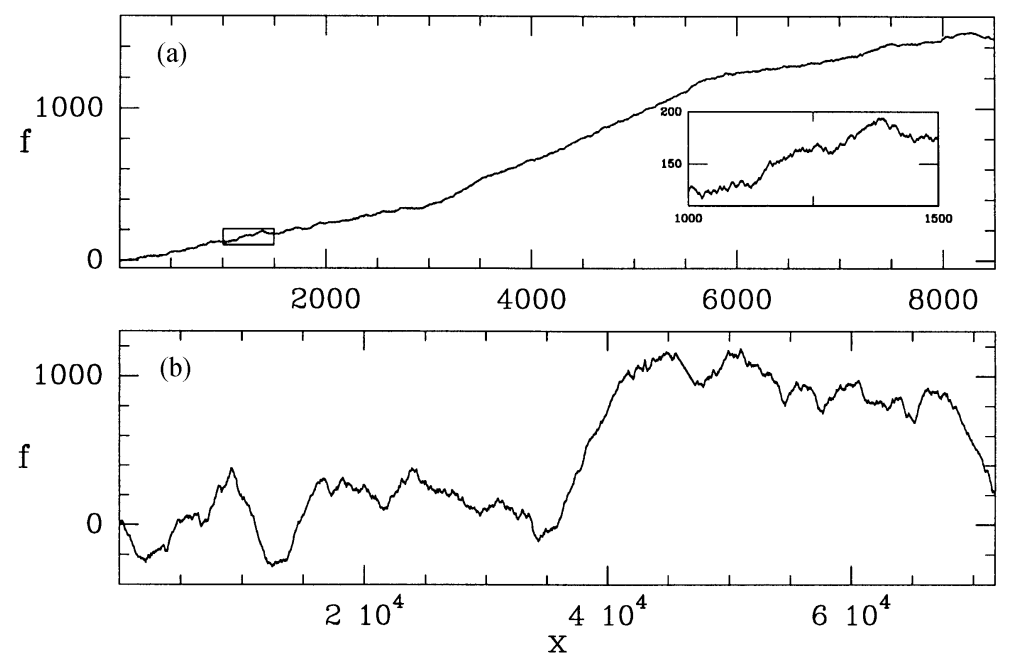

Fig. 4. The "DNA walk" representation of genomic sequences based on the purine-pyrimidine distinction. (a) The human desmoplakin I CDS $(L=8499)$. (b) The largest intron $(L=71718)$ in the human retinoblastoma susceptibility gene.

\subsection{DNA sequences}

The possible relevance of scale invariance and fractal concepts to the structural complexity of genomic sequences is the subject of considerable increasing interest [22]. During the past few years, there has been intense discussion about the existence, the nature and the origin of long-range correlations in DNA sequences. Different techniques including mutual information functions [119], autocorrelation functions [120,121], power spectra [122], "DNA walk" representation [21,22], Zipf analysis $[123,124]$ were used for statistical analysis of DNA sequences. But despite the effort spent, there is still some continuing debate on rather struggling questions. In that respect, it is of fundamental importance to corroborate the fact that the reported longrange correlations are not just an artefact of the compositional heterogeneity of the genome organization [120,125-128]. Furthermore, it is still an open question whether the long-range correlation properties are different for protein-coding (exonic) and noncoding (intronic, intergenic) sequences [21,22,119-124,129].

One of the main obstacles to long-range correlation analysis is the mosaic structure of DNA sequences which are well known to be formed of "patches" ("strand bias") of different underlying compositions [130-132]. As illustrated in Fig. 4, these patches appear as trends in the "DNA walk" landscapes and are likely to introduce some breaking of scale invariance [125-128]. Most of the technique used so far for characterizing the presence of long-range correlations are not well adapted to study patchy sequences. In a previous work [133], we have emphasized the wavelet transform (WT) as a very powerful technique for fractal analysis of DNA sequences. By taking advantage of the freedom in the choice of the analyzing wavelet, one can use wavelets that oscillate 
enough in order to make the WT microscope blind to low frequency trends. With these adequate filters one can actually reveal and quantify the scaling properties of the "DNA walks". Here we report on recent results obtained by applying the WTMM method to various genomic sequences selected in the human genome [134,135]. More precisely, 121 DNA sequences were selected with the requirement that their overall lengh $L \geqslant 2000$ nucleotides, so that the range of scales available to fractal scaling be large enough to make the analysis meaningful with respect to finite size effects. We took the sequences from EMBL data bank and processed seperately 47 coding (individual exons, CDS's) and 74 noncoding (individual introns) regions. To graphically portray these sequences we follow the so-called "DNA walk" analysis [21] which requires first to convert the four letter $(\mathrm{A}, \mathrm{C}, \mathrm{G}, \mathrm{T})$ text into a binary sequence. This can be done, for example, on the basis of purine $(A, G)$ vs pyrimidine $(C, T)$ distinction, by defining the incremental variable that associates to position $i$ the value $\chi(i)=1$ or -1 , depending on whether the $i$ th nucleotide of the sequence is a purine or a pyrimidine. (We refer the reader to Refs. [134,135] for similar analysis with the two complementary pair-base identifications). In Fig. 4 are illustrated the "DNA walk" graphs corresponding to a protein coding sequence, namely the human desmoplakin I CDS (Fig. 4a) and to a non coding sequence, namely the largest intron in the human retinoblastoma susceptibility gene (Fig. 4b). The patchiness of the coding sequence in Fig. 4a is patent; one clearly recognizes three regions of different bias. When applying the WTMM method to this coding "DNA walk" landscape, one gets a $\tau(q)$ spectrum (Eq. (13)) which remarkably falls on a straight line (the hallmark of homogeneous fractal signals) of slope $h=\partial \tau / \partial q=0.50 \pm 0.01$, provided the analyzing wavelet has at least two vanishing moments $\left(n_{\psi}=2\right)$ in order to be orthogonal to the observed linear trends [134]. A similar result is obtained for the noncoding sequence in Fig. 4b, except that the slope of the $\tau(q)$ spectrum $h=0.60 \pm 0.02$, is now significantly larger than $1 / 2$. These results are actually quite representative of the results obtained for our statistical sample of 47 coding and 74 noncoding sequences [134,135]. When averaging the partition functions over these two statistical samples, we get $\bar{Z}_{\mathrm{C}}(q, a)$ and $\bar{Z}_{\mathrm{NC}}(q, a)$ which both scale with the exponent predicted for homogeneous fractional Brownian motions, i.e., $\tau(q)=q H-1[28,29,134]$. The main difference which allows us to distinguish coding from noncoding sequences is the presence of long range correlations in the latter: $\bar{H}_{\mathrm{NC}}=0.59 \pm 0.02$, while the former look like uncorrelated random walks: $\bar{H}_{\mathrm{C}}=0.51 \pm 0.02[134,135]$.

One of the most striking results of our WTMM analysis is the fact that the $\tau(q)$ spectra extracted for the sets of exons and introns we have considered in the human genome, are surprisingly in remarkable agreement with the theoretical prediction for Gaussian processes. Within that prospect, we have studied the probability distribution function of wavelet coefficient values $P\left(T_{\psi^{(2)}}(., a)\right)$, as computed at a fixed scale $a$ in the fractal scaling range [134]. The distributions obtained for both the coding and noncoding DNA sequences of Fig. 4 are shown in Figs. 5a and 5b, respectively. When increasing the scale parameter $a$, the distributions become wider, but when plotting $\ln P$ vs $T_{\psi} / \sigma(a)$, where $\sigma(a)$ is the r.m.s value of $T_{\psi}$ at scale $a$, all the data computed 

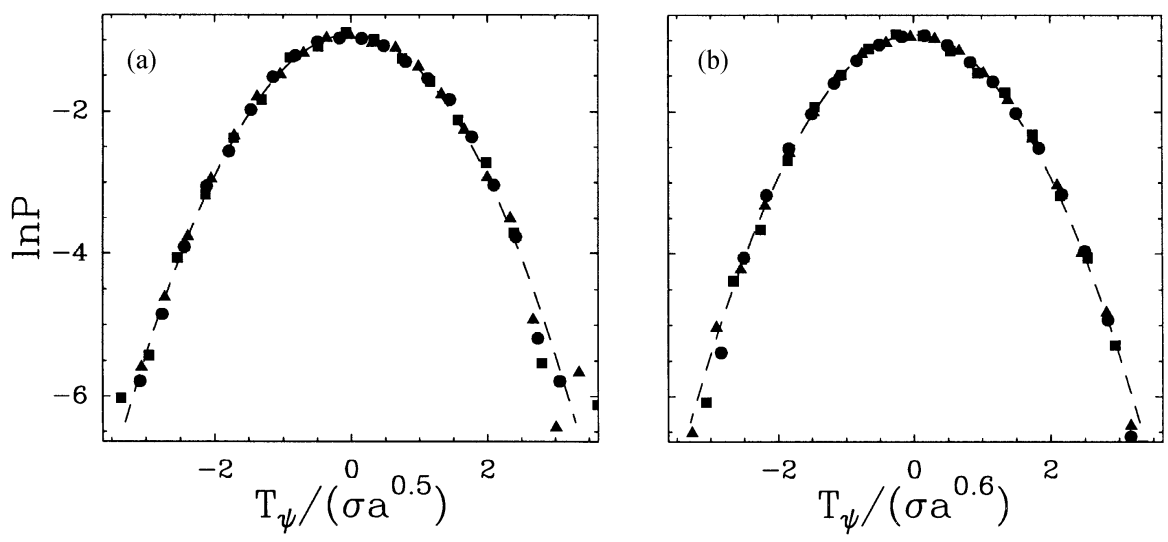

Fig. 5. Probability density functions of wavelet coefficient values at fixed scale $a=2^{2}(\bullet), a=2^{3}(\mathbf{\Delta})$, $a=2^{4}(\mathbb{\square})$ corresponding approximately to 32,64 and 128 nucleotides; the analyzing wavelet is the second derivative of the Gaussian function $\psi^{(2)}$. $\ln P$ is plotted vs $T_{\psi} / \sigma(a)$, where $\sigma(a)=\sigma a^{H}$ is the r.m.s. value. (a) Human desmoplakin I CDS sequence: $H=0.50$. (b) Largest intron in the human retinoblastoma susceptibility gene: $H=0.60$. The dashed lines are parabolas characteristic of Gaussian statistics.

at different scales fall on the same parabola independently of the nature of the sequence. Thus, as explored through the WT microscope, the basic fluctuations in "DNA walks" are likely to have Gaussian statistics. The presence of long-range correlations in the human introns is in fact contained in the scale dependence of $\sigma(a) \sim a^{H}$ where $H=0.60 \pm 0.02$ as compared to the uncorrelated random walk value $H=0.50 \pm 0.02$ obtained for the coding sequences [134].

In Fig. 6 are reported the results of a similar statistical WTMM analysis when classifying these DNA sequences into categories that correspond to a given GC content $[134,135]$. The idea of looking for a link between the long-range correlation properties and the GC content of the sequences results from the remark that the WTMM method indeed fails to distinguish a few introns from actual exons [134]. These introns with an exponent $H$ close to $1 / 2$, actually correspond to DNA sequences with a low GC content (from $31 \%$ to $36 \%$ ). When investigating the scaling behavior of $a^{1 / 2} Z(1, a) \sim a^{H-1 / 2}$ for our set of introns, one notices some significant tendancy of the curves to become steeper when continuously increasing the GC content [135]. The corresponding values of the roughness exponent $H$ are reported in Fig. 6a. $H$ clearly increases from values close to $1 / 2$ at low $\mathrm{GC}$ content $(\sim 30 \%)$ up to values significantly larger than 0.6 at high GC content $(>60 \%)$. In Fig. $6 \mathrm{a}$ are also shown the estimates of the roughness exponent for the coding sequences. Whether the CDS be poor or rich in GC, it does not seem to possess strong long-range correlations as indicated by an exponent $H$ close to $1 / 2$. Because of the "period three" codon structure of coding DNA, it is natural to investigate separately the three subsequences relative to the position $(1,2$ or 3) of the bases within their codons [136]. We have built up these subsequences from our 35 largest CDS sequences and we have repeated the WTMM analysis [135]. As shown in Fig. 6(b), for the first and the second subsequences, one gets results 


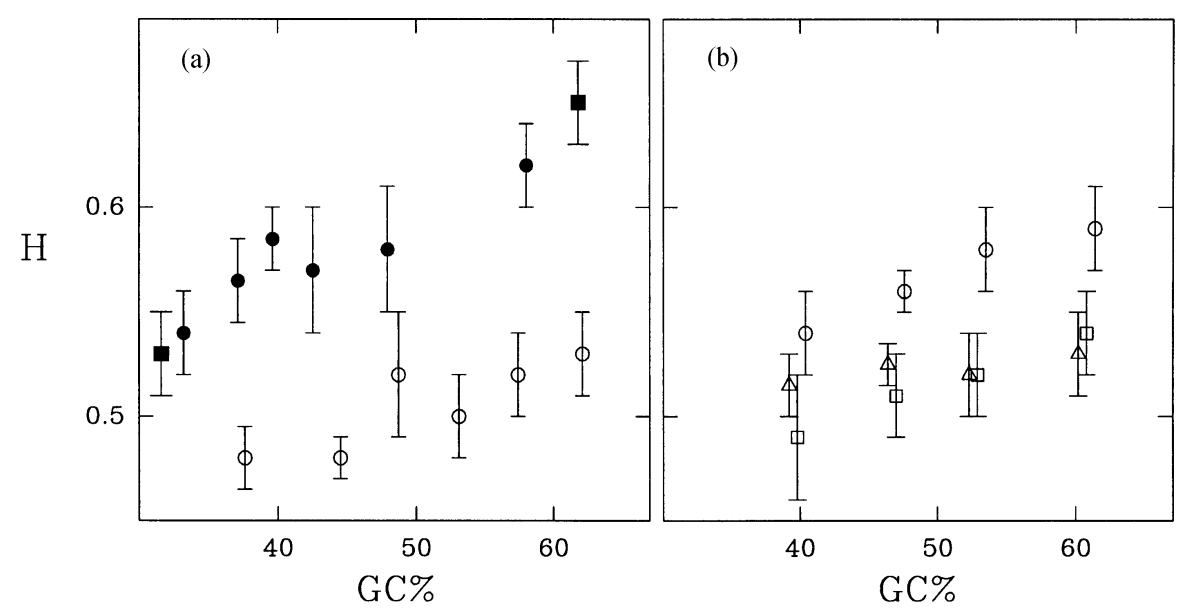

Fig. 6. WTMM estimate of the roughness exponent $H$ vs the GC content of the DNA sequence. (a) Introns: (•) $L \simeq 50000$, (ם) $L \simeq 15000$; CDSs: (o) $L \simeq 50000$. (b) Coding subsequences relative to position $1(\triangle), 2(\square)$ and $3(\circ)$ of the bases within the codons: $L \simeq 20000$. $L$ is the number of nucleotides involved in our WTMM statistical analysis.

quite consistent with the estimates obtained with the overall CDS sequences: whatever the GC content, the exponent $H$ does not significantly depart from the value $1 / 2$. (Note that the data do not exclude a possible slow increase of $H_{\mathrm{C} 2}$.) For the third subsequence, $H$ is found to increase up to values close to 0.60 at high GC content, which brings the clue that this subsequence exhibits [GC]-dependent long-range correlations very much like those observed in Fig. 6a for introns. Let us mention that we have checked that these observations do not result from the exon concatenation in the CDS's [135].

The results of our WTMM analysis clearly show that the GC content is likely to be relevant to the long-range correlation properties observed in both intronic and exonic DNA sequences. This observation is likely to be of fundamental importance since it suggests that the long-range correlations are related to the mechanisms involved in the "isochore structure" formation of the human genome [130-132,137]. The human genome is well known to be compartimentalized into wide $\left(\sim 10^{5}\right.$ nucleotides $)$ specific domains with uniform GC content, called isochores; appreciable scatter of the isochore GC content is actually observed when comparing different domains. Thanks to the functional constraints acting on coding sequences embedded in the GC rich regions, these non-equilibrium processes should be less active on exons, with a concomittant lack of long-range correlations as compared to the surrounding introns. Since these constraints are less stringent on the third base of the codons (most of the synonymous in the genetic code are due to the change of the third base in the codon), this would explain the correlations observed between these nucleotides in high GC containing exons. In human genes, the frequencies of the third base of codons are highly correlated with neighbouring intronic GC content [138]. This property favors the hypothesis that 
the exonic correlations are produced by the same mechanisms which lead to intronic correlations. It is likely that the observations reported here also extend to genomes of mammals and warmblooded vertebrates. The exploration of genomes of various organisms including unicellular eukaryotes and prokaryotes is currently under progress.

\section{Further developments and perspectives}

As we have just seen in the previous section, the application of wavelet analysis has already produced very promising results in various contexts. Based on this initial success, we anticipate significant theoretical and experimental developments during the next few years. In this section, we briefly describe some works currently in progress, which are likely to be the guidelines for future research.

Towards a "grand canonical" description of fractal signals. Even though Eq. (9) is not directly used for estimating the Hölder exponents, it is the cornerstone of the wavelet based multifractal formalism [28,29,33,60,92]. As this relation holds only for cusp-like singularities, this formalism is not valid if the fractal function $f$ involves other types of singularities. Let us consider for example the chirp function $[32,139,140]$ :

$$
f(x)=\left|x-x_{*}\right|^{h} \sin \left(\frac{1}{\left|x-x_{*}\right|^{\beta}}\right), \quad h>0, \beta>0 .
$$

This function is singular at $x=x_{*}$ and its Hölder exponent is $h\left(x_{*}\right)=h$. However, a direct estimate of this exponent using Eq. (9) would lead to a wrong result. Indeed, since the function $f(x)$ is infinitely oscillating around $x_{*}$, cancellations appear when this function is integrated against a smooth function, leading to a function more regular than expected. Such a singular behavior is referred to as an oscillating singularity $[32,139,140]$. Actually, contrary to functions with cusp singularities, the primitive of the oscillating function in Eq. (18) has an Hölder exponent $h+1+\beta \neq h+1$. Let us note that a cusp can be seen as an oscillating singularity with $\beta=0$. Thus, in order to fully characterize a singular behavior, one needs two exponents: the Hölder exponent $h$ and the oscillation exponent $\beta$ which characterizes the local power-law divergence of the instantaneous frequency [141]. In a recent work [142,143], we have elaborated on the theoretical foundations of a generalized WT multifractal formalism that describes statistically the fluctuations of both the Hölder exponent $h$ and the oscillation exponent $\beta$ of a fractal function. More specifically, this new formalism allows us to get the singularity spectrum $D(h, \beta)$ which corresponds to the Hausdorff dimension of the set of points $x$ corresponding to the same Hölder and oscillation exponents, i.e., $h(x)=h$ and $\beta(x)=\beta$. Whereas the partition function used in the classical formalism $[28,29,33,60,92]$ is indexed by a single parameter (conjugated to the Hölder exponent $h)$, this new description $[142,143]$ is based on a partition function involving two intensive parameters (associated to the exponents $h$ and $\beta$ ). In that sense, it is the analog of a "grand canonical" formalism whereas the classical formalism (Eq. (12)), can be identified to a "canonical" description. The application of this unified "grand canonical" 
description to experimental situations previously investigated with the WTMM method might occasionally lead to very suprising and therefore very interesting results.

Generalization of the WTMM method to multifractal image analysis. Ever since the explosive propagation of fractal ideas [1] throughout the scientific community in the late 1970s and early 1980s, there have been numerous applications to surface science. Indeed, a wide variety of natural and technical processes generate complex interfaces [3-7,9,11]. Assigning a fractal dimension to those irregular surfaces is now become routine in various fields including topography, defect and fracture studies, growth phenomena, erosion and corrosion processes and many other areas in physics, chemistry, biology, geology, meteorology and material sciences. But except some attempts to extend the structure function method to characterize the roughness fluctuations of selfaffine surfaces [144], there is a need of a powerful approach to multifractal analysis in 2D. Very recently, the WTMM method has been generalized to multifractal image analysis $[144,145]$. This generalization relies upon the extension of the WTMM representation in two dimensions, as proposed by Mallat and collaborators [32,146]. Note that this representation is actually strongly inspired from Canny's multiscale edge detectors [147] which are commonly used in computer vision. This isotropic 2D version of the WTMM methodology $[144,145]$ has been calibrated on deterministic self-similar interfaces and random self-affine surfaces (fractional Brownian surfaces and multifractal counter-parts). Preliminary applications to high-resolution satellite data and simulated radiance fields for stratocumulus clouds have brought a new light in the context of multifractal analysis of atmospheric data [145]. Future applications of this new numerical technology to rough surfaces that occur in various domains of fundamental as well as applied siences, look very promising.

From one-point to two-point statistics. The classical multifractal formalism [3443] as well as its wavelet based generalization [28,29,33,60,92], are nothing but a one-point statistical analysis which mainly consists in characterizing the way some pdf evolves when exploring the scales. Note that one can learn much more from the spacescale representation provided by the WT. In particular, one can proceed to a two-point statistical analysis by investigating correlations, not only at the same scale as before, but also across the scales. Such an analysis has been recently initiated in the context of fully developed turbulence [148] with the confirmation and even more the demonstration of the existence of a log-normal cascading process underlying the turbulent velocity fluctuations. More surprising are the results of a similar cross-correlation analysis of financial time-series [149]. Underlying the fluctuations of the volatility (standard deviation) of the price variations, there exists a causal information cascade from large to small time scales that can be visualized with the wavelet representation. Let us emphasize that the fact that variations of prices over a one month scale influence in the future the daily price variations, is likely to be extraordinarily rich in consequences and this, not only for the fundamental understanding of the nature of financial markets but also (and maybe more important) for practical applications. Indeed, the nature of the correlations across scales that are implied by this causal cascade has profound implications on the market risk, a problem of upmost concern for all financial institutions 
as well as individuals. There is no doubt in our minds that similar two-point statistical analysis will lead to significant progress in other fields than hydrodynamic turbulence and finance.

Solving the inverse fractal problem from wavelet analysis. The issue of carrying out a statistical mechanics of fractal objects has been mainly addressed in the context of dynamical system theory [36-40,43,52]. In particular, Feigenbaum [150] has shown that the microscopic information about a deterministic multiplicative dynamical system and its scaling properties is contained in the so-called scaling function which describes the scalings or contractions of the various elements of the attractor in time. This scaling function can be seen as the analog of the Hamiltonian. From the knowledge of this function, one can use the transfer matrix technique $[40,151,152]$ to compute the thermodynamic functions of interest, i.e., the partition function exponent $\tau(q)$ and the $D(h)$ singularity spectrum. On a more general ground, for any fractal object that can be observed in nature, there is a need to go beyond simple statistical averages and eventually to extract some "microscopic" information about their underlying hierarchical structure. In many cases, the self-similarity properties of fractal objects can be expressed in terms of a dynamical system which leaves the object invariant. The inverse problem consists in recovering this dynamical system (or its main characteristics) from the data representing the fractal object. This problem has been previously approached within the theory of Iterated Function Systems (IFS) [153-155]. But the methods developed in this context are based on the search of a "best fit" within a prescribed class of IFS attractors (mainly linear homogeneous attractors). In that sense, they approximate the self-similarity properties more than they reveal them. In a recent work $[82,156]$, we have shown that, in many situations, the space-scale representation provided by the WT of a fractal object can be used to extract some one-dimensional map which accounts for its construction process. More concretely, we have developed a waveletbased tree matching algorithm that provides a very efficient tool for solving the inverse fractal problem. This algorithm has been tested on discrete period-doubling dynamical systems and shown to reveal the renormalization operation which is essential to the understanding of the universal properties of this transition to chaos. One of the first and very promising applications of this new technique is the recent discovery [156-159] of a statistically predominant five-fold structural symmetry and of Fibonacci sequences associated with the branching of fractal aggregates generated by diffusion limited processes (examples include viscous fingering, electrochemical crystals, bacteria colonies, ...). We are convinced that further applications of this wavelet-based strategy for solving the inverse fractal problem will lead to similar major breakthroughs in various fields where multi-scale phenomena are ubiquitous.

\section{Acknowledgements}

We would like to thank Y. d'Aubenton-Carafa, P.V. Graves and C. Thermes for their collaboration to the DNA part of the work reported in this manuscript. We 
acknowledge very stimulating discussions with F. Argoul, J. Arrault, B. Castaing, Y. Couder, A. Davis, N. Decoster, S. Douady, B. Dubrulle, J. Elezgaray, Y. Gagne, F. Graner, A. Henaut, S. Jaffard, D. Sornette, P. Tabeling and H. Willaime. We are very grateful to Y. Gagne and Y. Malecot for the permission to use their experimental turbulent signals. This work was supported by "Direction des Recherches, Etudes et Techniques" under contract (DRET no 95/111), the GIP-GREG (project "Motifs dans les Séquences), the Ministère de l'Education Nationale, de l'Enseignement Supérieur, de la Recherche et de l'Insertion Professionnelle ACC-SV (project "Génétique et Environnement") and by NATO (Grant n CRG 960176).

\section{References}

[1] B.B. Mandelbrot, Fractals: Form, Chance and Dimension, Freeman, San Francisco, 1977; The Fractal Geometry of Nature, Freeman, San Franscisco, 1982.

[2] H.E. Stanley, N. Ostrowski (Eds.), On Growth and Form: Fractal and Non-Fractal Patterns in Physics, Martinus Nijhoff, Dordrecht, 1986; Random Fluctuations and Pattern Growth, Kluwer, Dordrecht, 1988.

[3] J. Feder, Fractals, Pergamon, New York, 1988.

[4] T. Vicsek, Fractal Growth Phenomena, World Scientific, Singapore, 1989.

[5] A. Aharony, J. Feder (Eds.), Fractals in Physics, Essays in honour of B.B. Mandelbrot, Physica D 38 (1989).

[6] F. Family, T. Viscek (Eds.), Dynamics of Fractal Surfaces, World Scientific, Singapore, 1991.

[7] T. Vicsek, M. Schlesinger, M. Matsushita (Eds.), Fractals in Natural Science, World Scientific, Singapore, 1994.

[8] B.J. West, W. Deering, Phys. Rep. 254 (1994) 1.

[9] G.G. Wilkinson, I. Kanellopoulos, J. Mégier (Eds.), Fractals in Geoscience and Remote Sensing, Image Understanding Research Series, vol. 1, ECSC-EC-EAEC, Brussels, Luxemburg, 1995.

[10] P. Tabeling, O. Cardoso (Eds.), Turbulence: A Tentative Dictionary, Plenum, New York, 1995.

[11] A.-L. Barabási, H.E. Stanley, Fractal Concepts in Surface Growth, Cambridge Univ. Press, Cambridge, 1995.

[12] B.B. Mandelbrot, J.W. Van Ness, SIAM Rev. 10 (1968) 422.

[13] H.O. Peitgen, D. Saupe (Eds.), The Science of Fractal Images, Springer, Berlin, 1987.

[14] B.B. Mandelbrot, J. Bus. Univ. Chicago 40 (1967) 393.

[15] B.B. Mandelbrot, H.M. Taylor, Oper. Res. 15 (1967) 1057.

[16] W. Li, Int. J. Bifurcation Chaos 1 (1991) 583.

[17] M. Goodchild, Math. Geol. 12 (1980) 85.

[18] B.J. West, Fractal Physiology and Chaos in Medecine, World Scientific, Singapore, 1990.

[19] F. Anselmet, Y. Gagne, E.J. Hopfinger, R.A. Antonia, J. Fluid Mech. 140 (1984) 63.

[20] Y. Gagne, Thesis, University of Grenoble, 1987.

[21] C.K. Peng, S.V. Buldyrev, A.L. Goldberger, S. Havlin, F. Sciortino, M. Simons, H.E. Stanley, Nature 356 (1992) 168.

[22] H.E. Stanley, S.V. Buldyrev, A.L. Goldberger, S. Havlin, S.M. Ossadnik, C.K. Peng, M. Simons, Fractals 1 (1993) 283.

[23] R.F. Voss, Physica D 38 (1989) 362; in Ref. [13], p. 21.

[24] B. Dubuc, J.F. Quiniou, C. Roque-Carmes, C. Tricot, S.W. Zucker, Phys. Rev. A 39 (1989) 1500.

[25] G.A. Edgard, Measure, Topology and Fractal Geometry, Springer, Berlin, 1990.

[26] U. Frisch, G. Parisi, in: M. Ghil, R. Benzi, G. Parisi (Eds.), Turbulence and Predictability in Geophysical Fluid Dynamics and Climate Dynamics, North-Holland, Amsterdam, 1985, p. 84.

[27] A.L. Barabási, T. Vicsek, Phys. Rev. A 44 (1991) 2730.

[28] J.F. Muzy, E. Bacry, A. Arneodo, Int. J. Bifurcation Chaos 4 (1994) 245.

[29] A. Arneodo, E. Bacry, J.F. Muzy, Physica A 213 (1995) 232.

[30] S. Jaffard, C.R. Acad. Sci. Paris 308 serie I (1989) 79; serie I (1992) 19.

[31] M. Holschneider, P. Tchamitchian, in Ref. [65], p. 102. 
[32] S. Mallat, W.L. Hwang, IEEE Trans. Inform. Theory 38 (1992) 617.

[33] E. Bacry, J.F. Muzy, A. Arneodo, J. Stat. Phys. 70 (1993) 635.

[34] R. Benzi, G. Paladin, G. Parisi, A. Vulpiani, J. Phys. A 17 (1984) 3521.

[35] E.B. Vul, Ya.G. Sinai, K.M. Khanin, J. Russ. Math. Surv. 39 (1984) 1.

[36] T.C. Halsey, M.H Jensen, L.P. Kadanoff, I. Procaccia, B.I. Shraiman, Phys. Rev. A 33 (1986) 1141.

[37] R. Badii, Thesis, University of Zurich, 1987.

[38] P. Collet, J. Lebowitz, A. Porzio, J. Stat. Phys. 47 (1987) 609.

[39] M.J. Feigenbaum, J. Stat. Phys. 46 (1987) 919, 925.

[40] M.H. Jensen, L.P. Kadanoff, I. Procaccia, Phys. Rev. A 36 (1987) 1409.

[41] G. Paladin, A. Vulpiani, Phys. Rep. 156 (1987) 148.

[42] B.B. Mandelbrot, Fractals and Multifractals: Noise, Turbulence and Galaxies, Selecta, vol. 1, Springer, New York, 1989.

[43] D. Rand, Ergod Th. and Dyn. Sys. 9 (1989) 527.

[44] A. Bunde, S. Havlin (Eds.), Fractals and Disordered Systems, Springer, Berlin, 1991.

[45] P. Meakin, in: C. Domb, J.L. Lebowitz (Eds.), Phase Transition and Critical Phenomena, vol. 12, Academic Press, Orlando, 1988, p. 355.

[46] B.B. Mandelbrot, J. Fluid. Mech. 62 (1974) 331.

[47] U. Frisch, S.A. Orzag, Turbulence: Challenges for Theory and Experiments, Physics Today (1990) 24.

[48] C. Meneveau, K.R. Sreenivasan, J. Fluid Mech. 224 (1991) 429.

[49] Ya.G. Sinai, J. Russ. Math. Surveys 27 (1972) 21.

[50] R. Bowen, Lect. Notes. in Maths 470 (1975) 1.

[51] D. Ruelle, Thermodynamic Formalism, Addison-Wesley, Reading, MA, 1978.

[52] T. Bohr, T. Tèl, in: B.L. Hao (Ed.), Direction in Chaos, vol. 2, World Scientific, Singapore, 1988.

[53] A. Renyi, Probability Theory, North-Holland, Amsterdam, 1970.

[54] P. Grassberger, Phys. Lett. A 97 (1983) 227.

[55] H.G.E. Hentschel, I. Procaccia, Physica D 8 (1983) 435.

[56] P. Grassberger, I. Procaccia, Physica D 13 (1984) 34.

[57] P. Grassberger, R. Badii, A. Politi, J. Stat. Phys. 51 (1988) 135.

[58] U. Frisch, Turbulence, Cambridge Univ. Press, Cambridge, 1995.

[59] A.S. Monin, A.M. Yaglom, Statistical Fluid Mechanics, vol. 2, MIT Press, Cambridge, MA, 1975.

[60] J.F. Muzy, E. Bacry, A. Arneodo, Phys. Rev. E 47 (1993) 875; in Ref. [70], p. 323.

[61] P. Goupillaud, A. Grossmann, J. Morlet, Geoexploration 23 (1984) 85.

[62] A. Grossmann, J. Morlet, SIAM J. Math. Anal. Appl. 15 (1984) 723; in: L. Steit (Ed.), Mathematics and Physics, Lecture on Recent Results, World Scientific, Singapore, 1985, p. 135.

[63] J.M. Combes, A. Grossmann, P. Tchamitchian (Eds.), Wavelets, Springer, Berlin, 1989.

[64] Y. Meyer, Ondelettes, Hermann, Paris, 1990.

[65] P.G. Lemarié (Ed.), Les Ondelettes en 1989, Springer, Berlin, 1990.

[66] Y. Meyer (Ed.), Wavelets and Applications, Springer, Berlin, 1992.

[67] I. Daubechies, Ten Lectures on Wavelets, SIAM, Philadelphia, 1992.

[68] M.B. Ruskai, G. Beylklin, R. Coifman, I. Daubechies, S. Mallat, Y. Meyer, L. Raphael (Eds.), Wavelets and Their Applications, Jones and Bartlett, Boston, 1992.

[69] C.K. Chui, An Introduction to Wavelets, Academic Press, Boston, 1992.

[70] Y. Meyer, S. Roques (Eds.), Progress in Wavelet Analysis and Applications, Editions Frontières, Gif sur Yvette, 1993.

[71] A. Arneodo, F. Argoul, E. Bacry, J. Elezgaray, J.F. Muzy, Ondelettes, Multifractales et Turbulences: de l'ADN aux croissances cristallines, Diderot Editeur, Arts et Sciences, Paris, 1995.

[72] G. Erlebacher, M.Y. Hussaini, L.M. Jameson (Eds.), Wavelets: Theory and Applications, Oxford Univ. Press, Oxford, 1996.

[73] M. Holschneider, Wavelets: An Analysis Tool, Oxford Univ. Press, Oxford, 1996.

[74] M. Holschneider, J. Stat. Phys. 50 (1988) 963; Thesis, University of Aix-Marseille II, 1988.

[75] A. Arneodo, G. Grasseau, M. Holschneider, Phys. Rev. Lett. 61 (1988) 2281; in Ref. [63], p. 182.

[76] A. Arneodo, F. Argoul, J. Elezgaray, G. Grasseau, in: G. Turchetti (Ed.), Nonlinear Dynamics, World Scientific, Singapore, 1989, p. 130.

[77] F. Argoul, A. Arneodo, G. Grasseau, Y. Gagne, E.J. Hopfinger, U. Frisch, Nature 338 (1989) 52.

[78] A. Arneodo, F. Argoul, G. Grasseau, in Ref. [65], p. 125. 
[79] F. Argoul, A. Arneodo, J. Elezgaray, G. Grasseau, R. Murenzi, Phys. Lett. A 135 (1989) 327; Phys. Rev. A 41 (1990) 5537.

[80] A. Arneodo, F. Argoul, E. Bacry, J. Elezgaray, E. Freysz, G. Grasseau, J.F. Muzy, B. Pouligny, in Ref. [66], p. 286.

[81] A. Arneodo, F. Argoul, E. Freysz, J.F. Muzy, B. Pouligny, in Ref. [68], p. 241.

[82] A. Arneodo, E. Bacry, J.F. Muzy, Europhys. Lett. 25 (1994) 479.

[83] A. Arneodo, in Ref. [72], p. 349.

[84] G. Grasseau, Thesis, University of Bordeaux I, 1989.

[85] E. Bacry, Thesis, University of Paris VII, 1992.

[86] J.F. Muzy, Thesis, University of Nice, 1993.

[87] D. Sornette, in: B. Dubrulle, F. Graner, D. Sornette (Eds.), Scale Invariance and Beyond, EDP Sciences \& Springer, Les Vlis \& Berlin, 1997, p. 235.

[88] E. Bacry, A. Arneodo, U. Frisch, Y. Gagne, E.J. Hopfinger, in: M. Lesieur, O. Metais (Eds.), Turbulence and Coherent Structures, Kluwer, Dordrecht, 1991, p. 203.

[89] M. Vergassola, R. Benzi, L. Biferale, D. Pissarenko, J. Phys. A 26 (1993) 6093.

[90] M. Vergassola, U. Frisch, Physica D 54 (1991) 58.

[91] J.D. Farmer, E. Ott, J.A. Yorke, Physica D 7 (1983) 153.

[92] J.F. Muzy, E. Bacry, A. Arneodo, Phys. Rev. Lett. 67 (1991) 3515.

[93] S. Jaffard, Multifractal formalism for functions: Part I and Part II, SIAM J. Math. Anal., to appear.

[94] A. Arneodo et al., Europhys. Lett. 34 (1996) 411.

[95] A.N. Kolmogorov, C. R. Acad. Sci. USSR 30 (1941) 301.

[96] B. Castaing, Y. Gagne, E.J. Hopfinger, Physica D 46 (1990) 177.

[97] P. Tabeling, G. Zocchi, F. Belin, J. Maurer, H. Willaime, Phys. Rev. E 53 (1996) 1613.

[98] S.G. Roux, Thesis, University of Aix-Marseille II, 1996.

[99] A. Arneodo, E. Bacry, J.F. Muzy, in: J.P. Bonnet, M.N. Glauser (Eds.), Eddy Structure Identification in Free Turbulent Shear Flows, Kluwer, Dordrecht, 1993, p. 153; in Ref. [10], p. 139.

[100] R. Benzi, S. Ciliberto, R. Tripiccione, C. Baudet, F. Massaioli, S. Succi, Phys. Rev. E 48 (1993) R29; Phys. Rev. E 51 (1995) 2672.

[101] R. Benzi, S. Ciliberto, C. Baudet, G.R. Chavarria, R. Tripiccione, Europhys. Lett. 24 (1993) 275.

[102] A.N. Kolmogorov, J. Fluid Mech. 13 (1962) 82.

[103] A.M. Obukhov, J. Fluid Mech. 13 (1962) 77.

[104] A. Arneodo, J.F. Muzy, S.G. Roux, J. Phys. II France 7 (1997) 363.

[105] A. Arneodo, S. Manneville, J.F. Muzy, S.G. Roux, Experimental evidence for anomalous scale dependent cascading process in turbulent velocity statistics, preprint, 1997.

[106] Z.S. She, E. Leveque, Phys. Rev. Lett. 72 (1994) 336.

[107] Z.S. She, E.C. Waymire, Phys. Rev. Lett. 74 (1995) 262.

[108] B. Dubrulle, Phys. Rev. Lett. 73 (1994) 959; J. Phys. II France 6 (1996) 1825.

[109] I. Daubechies, A. Grossmann, Y. Meyer, J. Math. Phys. 27 (1986) 1271.

[110] B. Castaing, Y. Gagne, M. Marchand, Physica D 68 (1993) 387.

[111] A. Naert, L. Puech, B. Chabaud, J. Peinke, B. Castaing, B. Hebral, J. Phys. II France 4 (1994) 215.

[112] B. Chabaud, A. Naert, J. Peinke, F. Chillà, B. Castaing, B. Hebral, Phys. Rev. Lett. 73 (1994) 3227.

[113] B. Castaing, B. Dubrulle, J. Phys. II France 5 (1995) 895.

[114] B. Castaing, J. Phys. II France 6 (1996) 105.

[115] F. Chillà, J. Peinke, B. Castaing, J. Phys. II France 6 (1996) 455.

[116] B. Castaing, in: B. Dubrulle, F. Graner, D. Sornette (Eds.), Scale Invariance and Beyond, EDP Sciences \& Springer, Les Vlis \& Berlin, 1997, p. 225.

[117] E.A. Novikov, Phys. Fluids A 2 (1990) 814; Phys. Rev. E 50 (1995) 3303.

[118] B. Dubrulle, in: B. Dubrulle, F. Graner, D. Sornette (Eds.), Scale Invariance and Beyond, EDP Sciences \& Springer, Les Vlis \& Berlin, 1997, p. 275.

[119] W. Li, Int. J. Bifurcation Chaos 2 (1993) 137.

[120] M.Ya. Azbel, Phys. Rev. Lett. 75 (1995) 168.

[121] H. Herzel, I. Große, Physica A 216 (1995) 518.

[122] R.F. Voss, Phys. Rev. Lett. 68 (1992) 3805; Fractals 2 (1994) 1.

[123] R.N. Mantegna, S.V. Buldyrev, A.L. Goldberger, S. Havlin, C.K. Peng, M. Simons, H.E. Stanley, Phys. Rev. E 52 (1995) 2939. 
[124] S. Havlin, S.V. Buldyrev, A.L. Goldberger, R.N. Mantegna, C.K. Peng, M. Simons, H.E. Stanley, Fractals 3 (1995) 269.

[125] S. Nee, Nature 357 (1992) 450.

[126] C.A. Chazidimitriou-Dreismann, L. Larhammar, Nature 361 (1993) 212.

[127] S. Karlin, V. Brendel, Science 259 (1993) 677.

[128] B. Borstnick, D. Pumpernik, D. Lukman, Europhys. Lett. 23 (1993) 389.

[129] S.V. Buldyrev, A.L. Goldberger, S. Havlin, R.N. Mantegna, M.E. Matsa, C.K. Peng, M. Simons, H.E. Stanley, Phys. Rev. E 51 (1995) 5084.

[130] G. Bernardi, B. Olofsson, J. Filipski, M. Zerial, J. Salinas, F. Cuny, M. Meunier-Rotival, F. Rodier, Science 228 (1985) 953.

[131] G.A. Churchill, Bull. Math. Biol. 51 (1989) 79.

[132] J.W. Fickett, D.C. Torney, D.R. Wolf, Genomics 13 (1992) 1056.

[133] A. Arneodo, E. Bacry, P.V. Graves, J.F. Muzy, Phys. Rev. Lett. 74 (1995) 3293.

[134] A. Arneodo, Y. D’Aubenton-Carafa, E. Bacry, P.V. Graves, J.F. Muzy, C. Thermes, Physica D 96 (1996) 291.

[135] A. Arneodo, Y. D’Aubenton-Carafa, B. Audit, E. Bacry, J.F. Muzy, C. Thermes, Eur. Phys. J. B 1 (1998) 259.

[136] P. Allegrini, M. Barbi, P. Grigolini, B.J. West, Phys. Rev. E 52 (1995) 5281.

[137] G. Bernardi, Annu. Rev. Genet. 23 (1989) 637.

[138] P. Sharp, G. Matassi, Current Opinion Gent. Dev. 4 (1994) 851.

[139] S. Jaffard, Y. Meyer, Wavelets methods for pointwise regularity and local oscillations of functions, Mem. A.M.S., to appear.

[140] A. Arneodo, E. Bacry, J.F. Muzy, Phys. Rev. Lett. 74 (1995) 4823.

[141] N. Delprat, B. Escudié, P. Guillemain, R. Kronland-Martinet, P. Tchamitchian, B. Torrésani, IEEE Trans. Inform. Theory 38 (1992) 644.

[142] A. Arneodo, E. Bacry, S. Jaffard, J.F. Muzy, J. Stat. Phys. 87 (1997) 179.

[143] A. Arneodo, E. Bacry, S. Jaffard, J.F. Muzy, Singularity spectrum of multifractal functions involving oscillating singularities, J. Fourier Anal. Appl. (1997), to appear.

[144] J. Arrault, Thesis, University of Bordeaux I, 1995.

[145] J. Arrault, A. Arneodo, A. Davis, A. Marshak, Phys. Rev. Lett. 79 (1997) 75.

[146] S. Mallat, S. Zhong, IEEE Trans. Patt. Anal. Machine Intell. 14 (1992) 710.

[147] J. Canny, IEEE Trans. Pattern Anal. Machine Intell. 8 (1986) 679.

[148] A. Arneodo, S. Manneville, J.F. Muzy, Phys. Rev. Lett. 80 (1998) 708.

[149] A. Arneodo, J.F. Muzy, D. Sornette, Causal cascade in the stock market from the infrared to the ultraviolet, Eur. Phys. J. B 1 (1998), to appear.

[150] M.J. Feigenbaum, J. Stat. Phys. 21 (1979) 669; Los Alamos Sci. 1 (1980) 4; Comm. Math. Phys. 77 (1980) 65.

[151] M.J. Feigenbaum, M.H. Jensen, I. Procaccia, Phys. Rev. Lett. 57 (1986) 1503.

[152] A.B. Chhabra, R.V. Jensen, K.R. Sreenivasan, Phys. Rev. A 40 (1989) 4593.

[153] M.F. Barnsley, S.G. Demko, Proc. R. Soc. London A 399 (1985) 243.

[154] M.F. Barnsley, Fractal Everywhere, Academic Press, New York, 1988.

[155] C.R. Handy, G. Mantica, Physica D 43 (1990) 17.

[156] A. Arneodo, F. Argoul, E. Bacry, J.F. Muzy, M. Tabard, Fractals 1 (1993) 629.

[157] A. Arneodo, F. Argoul, E. Bacry, J.F. Muzy, M. Tabard, Phys. Rev. Lett. 68 (1992) 3456; J. Diff. Eq. Appl. 1 (1995) 117.

[158] A. Arneodo, F. Argoul, J.F. Muzy, M. Tabard, Phys. Lett. A 171 (1992) 31; Physica A 188 (1992) 217.

[159] A. Kuhn, F. Argoul, J.F. Muzy, A. Arneodo, Phys. Rev. Lett. 73 (1994) 2998. 